\title{
Immediate-early gene transcriptional activation in hippocampus CA1 and CA3 does not accurately reflect rapid, pattern completion-based retrieval of context memory
}

\author{
Aleksandr Pevzner ${ }^{1,2}$ and John F. Guzowski ${ }^{1}$ \\ ${ }^{1}$ Center for the Neurobiology of Learning and Memory; Department of Neurobiology and Behavior; University of California, \\ Irvine, California 92697-3800, USA
}

\begin{abstract}
No studies to date have examined whether immediate-early gene (IEG) activation is driven by context memory recall. To address this question, we utilized the context preexposure facilitation effect (CPFE) paradigm. In CPFE, animals acquire contextual fear conditioning through hippocampus-dependent rapid retrieval of a previously formed contextual representation. Despite differences in behavior, we did not find any difference in CA1 or CA3 IEG activity associated with this rapid recall phase when comparing context preexposed and non-pre-exposed groups. These findings indicate that IEG activation in CA1 and CA3 is not an accurate readout of the neural activity associated with hippocampus-dependent rapid memory retrieval.
\end{abstract}

The hippocampus is posited to be part of the primary circuit involved in encoding and retrieval of context memory (Hirsh 1974; Fanselow 2000; Maren 2001; Rudy et al. 2004). Theories of hippocampal function propose that memory retrieval depends on neural reactivation of previously active ensembles within this region (Morris et al. 1977; Teyler and DiScenna 1986; Norman and O'Reilly 2003; Rolls and Kesner 2006; Teyler and Rudy 2007). This tenet has recently been tested with optogenetic stimulation and inhibition (Liu et al. 2012; Ramirez et al. 2013; Tanaka and Wiltgen 2013). While the experimental data are in accord with the theoretical proposal that retrieval of a context memory is dependent on reactivation within the hippocampus, so far the underlying mechanisms and accompanying neural activity that is associated with the retrieval process have not been addressed.

One proposed mechanism of memory retrieval in the hippocampus is through "replay" activity during sharp wave ripple events (SWRs) (Kudrimoti et al. 1999; Carr et al. 2011). SWRs are intermittent oscillatory patterns of network activity in the 150$200 \mathrm{~Hz}$ range. During these periods CA1 pyramidal cells fire synchronously in a pattern reflecting the activity of place cells which were recorded during a previous spatial exploration (Jones and Wilson 2005; Foster and Wilson 2006; Diba and Buzsáki 2007). This experience-dependent place cell synchronization during SWR is associated with memory recall and its replay is necessary for memory retrieval (Girardeau et al. 2009; Jadhav et al. 2012; Pfieffer and Foster 2013). In contrast to the electrophysiological data, the molecular correlates of retrieval-based neural activity are unknown. Thus, we tested if rapid retrieval of context memory would drive immediate-early gene (IEG) expression in the main output pathway of hippocampus, the CA1 subfield.

IEG imaging has been used extensively to measure hippocampal network activity in response to exposure to an environmental context (Guzowski et al. 1999, 2006; Barot et al. 2009;

\footnotetext{
${ }^{2}$ Present address: Center for Neuroscience, University of California Davis, Davis, CA 95618, USA

Corresponding author: jguzowsk@uci.edu

Article is online at http://www.learnmem.org/cgi/doi/10.1101//m.035469.114.
}

Miyashita et al. 2009; Wiltgen et al. 2010; Nalloor et al. 2012; Nomura et al. 2012; Pevzner et al. 2012). However, despite the broad use of IEGs to visualize activated ensembles, the design of past behavioral studies has precluded specifying the transcriptional activation of IEGs explicitly to either the encoding or retrieval of a context memory. A "retrieval" test does not circumvent this problem, as it is likely that the animal encodes (or "reencodes") the context during the test session (Tolman 1925; Tolman et al. 1946; Good et al. 1998). Thus, a task capable of disambiguating encoding and retrieval of context is required in order to map IEG activation to one or both of these neural processes.

Here we used the context preexposure facilitation effect (CPFE) paradigm (Matus-Amat et al. 2004; Rudy et al. 2004) to test whether hippocampal IEG expression is driven by rapid retrieval of context memory. The power of CPFE to dissociate encoding and retrieval processes stems from the finding that preexposure to a context alleviates the immediate-shock deficit (ISD). The ISD describes the observation that an animal shocked immediately upon placement into a novel chamber during training fails to develop conditioned freezing to that context as assessed during retention testing (Blanchard et al. 1976; Fanselow 1986). It is hypothesized that the ISD arises from inadequate time given to the animal to form a contextual representation prior to the shock (Fanselow 1990). The ISD can be overcome, however, by preexposing an animal to the chamber $24 \mathrm{~h}$ before the shock (Fanselow 1990; Kiernan and Westbrook 1993; Westbrook et al. 1994). This phenomenon, along with supporting studies, led to the hypothesis that a hippocampus-dependent representation of the context is established during the preexposure phase (Fanselow 1990; Barrientos et al. 2002; Matus-Amat et al. 2004), which can then be rapidly retrieved and associated with the shock during the immediate-shock phase (Barrientos et al. 2002; Rudy et al.

(C) 2014 Pevzner and Guzowski This article is distributed exclusively by Cold Spring Harbor Laboratory Press for the first 12 months after the full-issue publication date (see http://learnmem.cshlp.org/site/misc/terms.xhtml). After 12 months, it is available under a Creative Commons License (AttributionNonCommercial 4.0 International), as described at http://creativecommons .org/licenses/by-nc/4.0/. 
2002; Matus-Amat et al. 2007). Because the interval during the immediate-shock phase is too brief for the animal to encode the context de novo (Fanselow 1990; Wiltgen et al. 2011; Pevzner et al. 2012), comparing context preexposed and non-pre-exposed rats allows one to assess hippocampal network activity during the rapid recall of a context-specific memory.

Neuronal ensembles activated by two discrete behavioral experiences, such as two context exposures, can be visualized using Arc/Homer catFISH. Using this IEG imaging method, the first epoch is evaluated with Homer 1a expression while the second experience is visualized with Arc (Guzowski 2002; Vazdarjanova and Guzowski 2004; Kubik et al. 2007). Colocalization of the two IEG RNAs can be interpreted as activation of the same neuronal population, while nonoverlap in their expression is thought to arise from activation of two distinct populations.

Here we assessed the contribution of memory retrieval to IEG activation by combining the CPFE paradigm with Arc/Homer catFISH imaging. The CPFE paradigm is composed of three stages, each separated by $24 \mathrm{~h}$ : preexposure to an environmental context, immediate shock, and test of fear memory. During the immediateshock phase, animals engage in rapid retrieval of a contextual representation established during the preexposure (Barrientos et al. 2002; Rudy et al. 2002; Matus-Amat et al. 2007). If no contextual representation exists (i.e., no preexposure), then animals are unable to display conditioned fear during the memory test. We compared conditioning of rats given one of three different preexposure conditions. In the PE-A group, rats were given three brief preexposures (PE) to context A (a standard conditioning chamber; Coulbourn Instruments), the to-be shocked context, across three consecutive days. The first exposure was $5 \mathrm{~min}$, while the other two context exposures were $1 \mathrm{~min}$ each. Animals in the PE-B group were given equivalent preexposures to a different context (context B; an enclosed circular arena in a different room). The non-PE animals received no preexposure to any context and served to establish baseline freezing due to the immediate-shock training.

An ANOVA comparison of freezing (as defined in Fanselow 1982) during the 24 -h retention test revealed significant differences between the groups, with a Fisher's post hoc test confirming that the PE-A group froze significantly more than both PE-B and non-PE groups (Fig. 1A). Importantly, there was no difference in freezing between the PE-B and non-PE groups. In addition to the freezing data, the number of rears (Carrive 2000; Lever et al. 2006) was significantly different between the groups as determined using a Kruskal-Wallis test (Fig. 1B). Subsequent analysis using the Mann-Whitney $U$-test indicated that while the PE-A group reared less than the non-PE group, the comparison to the PE-B group approached, but did not reach, statistical significance $(U=11.5, P=0.051)$. More important, however, the PE-B group did not differ from the non-PE group in rears. This is consistent with the notion that the observed fear behavior in the PE-A group was not a product of generalized contextual fear conditioning, but rather resulted from an association of a specific contextual representation with shock. Both measures of fear behavior were strongly convergent, and illustrate that during the preexposure the rats formed a contextual representation of context $\mathrm{A}$, which was then rapidly retrieved during a subsequent brief exposure to support conditioning.

Given that animals in the PE-A group engaged in the rapid recall of a context-specific memory, we next examined hippocampal IEG activation during this brief context exposure. Analogous to the behavioral experiment, on the test day rats that were given preexposure to context A were placed back into the context for $5 \mathrm{sec}$. During this brief time, animals rapidly retrieve the cellular representation of the previously explored context (Rudy et al. 2002) through a pattern completion-like process requiring the dorsal hippocampus (Rudy and O'Reilly 1999, 2001; MatusAmat et al. 2004). Pattern completion, as it is applied to neural networks, is a computational process by which a partial input is able to result in the activation of the full network (Rudy and O'Reilly 1999). The brief exposure during the immediate-shock phase of CPFE is likened to a "partial input" because this interval is too brief for the animals to fully encode the context (Fanselow 1990; Pevzner et al. 2012). However, with pattern completion the partial input is capable of recalling the previously acquired context representation established during the preexposure phase (i.e., full network). In order to assess context specificity of the recalled network, animals were given a second, 5-min exposure to the environment $25 \mathrm{~min}$ after the first exposure. If IEG transcription is driven within the same neuronal ensemble by rapid memory retrieval (first behavioral epoch) and during ongoing experience (encoding or reencoding in the second behavioral epoch), then we predict to observe a high degree of colocalization of Arc and Homer RNAs in a subset of hippocampal neurons (Vazdarjanova and Guzowski 2004; Guzowski et al. 2005).

We tested two main hypotheses regarding the impact of context preexposure on cell activity: (1) it would decrease the behavioral experience needed for robust IEG activation in CA1 (Pevzner et al. 2012) and (2) it would increase context specificity in ensemble activation as assessed with catFISH in CA1 and CA3. The first hypothesis stems from a recent finding that increased exposure to a context was associated with an increase in Arc + cells in CA1 and the formation of a contextual representation (Pevzner et al. 2012). Thus, we reasoned the relationship of greater CA1 activity and formation of a context memory would also be preserved in the preexposed rats (PE-A), and this would be observed as an increase in IEG (Homer 1a) expression relative to non-PE rats. The 
second hypothesis was largely driven by the theoretical proposal that pattern completion during the rapid retrieval instantiates the full, previously acquired contextual representation.

At the termination of the experiment brains were processed for Arc/Homer 1 a catFISH (Vazdarjanova and Guzowski 2004). In CA1 there was a main group effect for percent Homer+ cells, which corresponds to activity from the first context exposure (Fig. 2A,B). While post hoc tests revealed that there was no difference between $\mathrm{PE}$ and non-PE groups, both groups were significantly different from the A/A group, which defined the maximal expected activity. The A/A group was not preexposed to the context, and on the test day was given two 5-min exposures to the same context. The lack of maximal Homer $1 a$ activation in CA1 with a brief context exposure is congruent with a past study demonstrating the same pattern of activity for Arc with brief context exposure (Pevzner et al. 2012). In contrast to Homer+ cells, there was no difference between the experimental groups for percent Arc+ cells (Fig. 2C). Importantly, all three context exposure groups demonstrated an increase in both Homer and Arc activity relative to the caged controls (CC) (Fig. 2B,C), indicating that

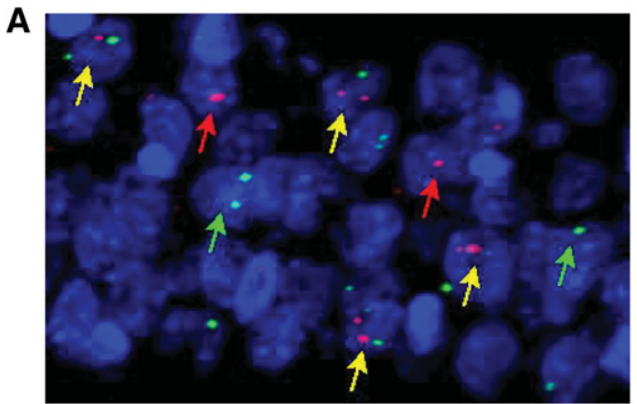

B

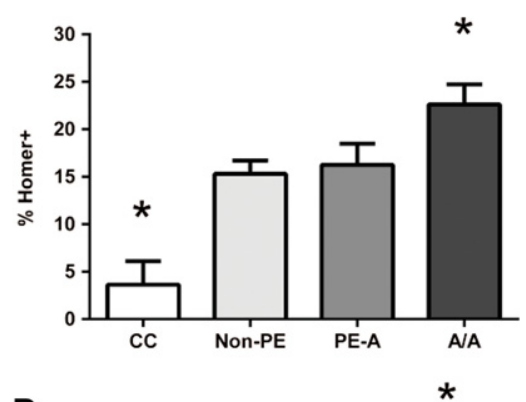

C

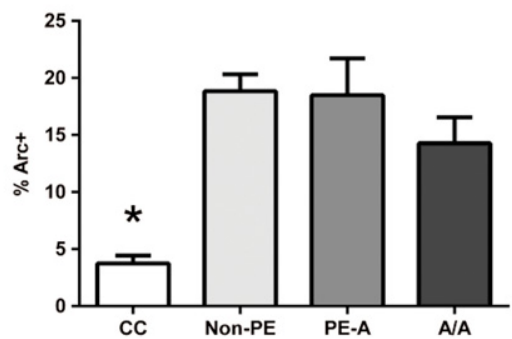

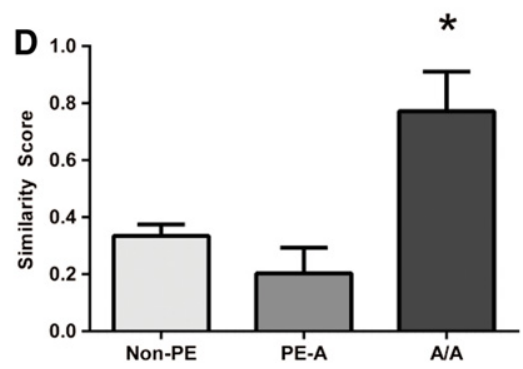

the detected IEGs resulted from neural activity associated with context exposure.

In order to test the context specificity of the IEG expression, we quantified the extent to which cells were active between both context exposures using a similarity score (SS) (Fig. 2D). This is a normalized measure that takes into account the level of IEG activity between the two epochs (Vazdarjanova and Guzowski 2004). Fisher's post hoc test showed that the SS of the A/A group was significantly greater from both the PE-A and non-PE groups. This difference was not due to more cells activated with a full 5-min exposure, because the SS metric accounts for total proportion of active cells. The higher SS for the A/A group indicates a greater degree of overlap in the neuronal ensembles activated during the two exposures to $\mathrm{A}$, as compared with the other groups. Surprisingly, preexposure did not increase overlap relative to non-pre-exposure, despite the fact that preexposure allowed animals to fear condition to the specific context (Fig. 1).

We next examined Arc/Homer expression in CA3. There were no overall differences in the percent of Homer+ or Arc+ cells between the three behavioral groups (Fig. 3A,B), consistent with an earlier study (Pevzner et al. 2012). Although preexposure had no effect on the proportion of active cells in CA3, it remained possible that prior experience would result in greater context-specific IEG activity, possibly due to a pattern completion-like process (Rudy and O'Reilly 1999). As in CA1, PE-A was not different from the non-PE group, while both had a significantly lower SS as compared with the A/A group (Fig. 3C). In sum, preexposure had no observable effect on transcriptional activation or context specificity of IEG expression in CA3. These IEG imaging results indicate that although animals engaged in rapid recall of a context-specific memory, this neural activity was not associated with altered levels or cellular patterns of IEG transcription.

In order to draw our main conclusion-that rapid memory retrieval does not drive IEG transcriptional activation in CA1 or CA3-it is necessary to evaluate the claim that the rats in fact engaged in recall of a specific context memory during the brief context exposure. First, the non-PE group displayed the classic immediate-shock deficit (ISD): high exploratory rearing and no conditioned freezing during the retention test (Fig. $1 \mathrm{~A}, \mathrm{~B})$. The lack of fear conditioning in the non-PE group also indicated that the observed fear response in the PE-A group was not due to animals conditioning to the transport to the experimental room (Rudy and O'Reilly 2001), as the non-PE group underwent the same transport procedure. While the non-PE group findings indicated that a previously established context memory is necessary for conditioning in CPFE, the PE-B control group attested to the specificity of the recalled context memory. It is presumed that the PE-B group animals formed a contextual representation of 

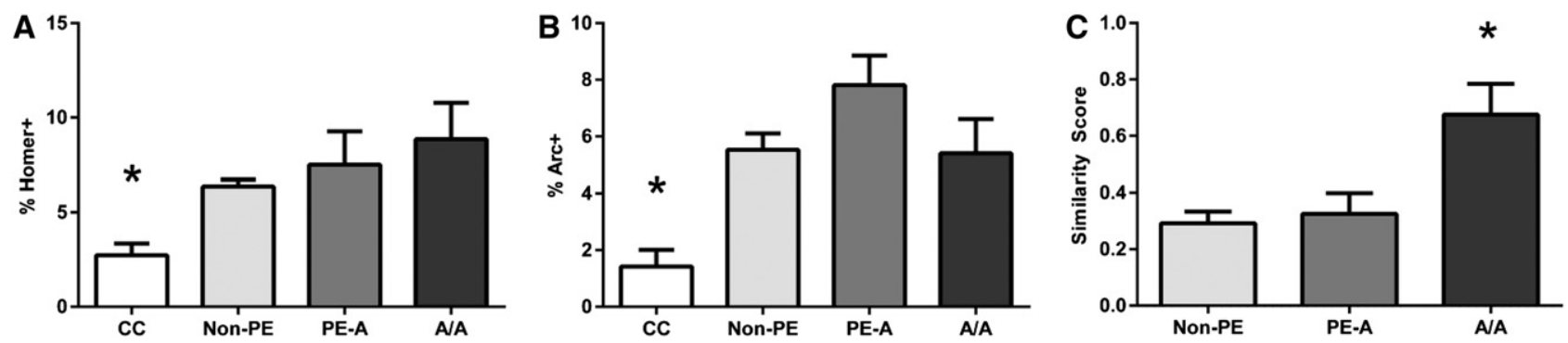

Figure 3. Context preexposure does not alter IEG expression to brief context reexposure in CA3 ensembles. ( $A$ ) Homer+ cells in CA3 expressed as a percent of the total neuronal population. Context exposure resulted in a significant increase of Homer + cells, relative to the caged control (CC, $n=$ 7) group (ANOVA: $F_{(3,20)}=7.18, P=0.002$; Fisher's post hoc: Non-PE $(n=6)$, PE-A $\left.(n=6), \mathrm{A} / \mathrm{A}(n=5) P<0.02\right)$. Brief exposure resulted in a similar proportion of active cells irrespective of preexposure condition $(P=0.194)$. Neither $P E-A$ nor Non-PE groups were different from $A / A(N o n-P E P=$ 0.132; PE-A $P=0.775)$. (B) ArC + cells in CA3 expressed as a percent of total population. The CC had significantly fewer $\operatorname{ArC}+$ cells $\left(F_{(3,20)}=10.64\right.$, $P=0.0002$; Non-PE, PE-A, A $/ A P<0.003$ ) There was no statistical difference between any context exposed groups in number of Arc+ cells. (C) Ensemble overlap in CA3 between the two context exposures. Prior to calculating the SS, the baseline (CC) IEG levels were subtracted from the other groups. Preexposure did not increase ensemble overlap in CA3 relative to non-pre-exposed animals $(P=0.753)$, however both groups were different from A/A group $\left(F_{(2,14)}=7.4, P=0.006\right)$; Non-PE $(P=0.003)$ PE-A $(P=0.006) .\left(^{*}\right) P<0.05$, different from all other groups.

context B, similar to the PE-A animals forming a representation of context A. However, the PE-B group did not display any conditioned fear to the shock-paired context (A) and was indistinguishable from the non-PE group. Thus, the PE-B control together with the non-PE group behaviorally confirmed that during the brief context exposure animals are only able to associate the context with a shock if they retrieve a previously acquired memory of that specific context.

To gain insight into the regulation of IEG expression during encoding or retrieval of context memory, it is necessary to understand the neural activity associated with these cognitive events. Interestingly, the cellular mechanisms engaged during encoding and retrieval of a context memory in CPFE have been shown to be distinct. Infusion of the NMDA receptor antagonist AP-5 into the dorsal hippocampus during the context preexposure phase (i.e., the encoding phase) impaired conditioning in CPFE (Fanselow 1990; Barrientos et al. 2002; Matus-Amat et al. 2004, 2007). This result implicates NMDA receptor-dependent plasticity in hippocampus as critical for the consolidation of a cellular representation of context acquired during preexposure. Consistent with this view, a recent study demonstrated that maximal induction of Arc, which is required for NMDA-dependent long-term potentiation in hippocampus (Messaoudi et al. 2007; Guzowski et al. 2000), was correlated with the formation of a contextual representation supporting fear memory (Pevzner et al. 2012).

With respect to the retrieval of context memory during the immediate-shock phase of CPFE, several studies implicate the hippocampus as the critical site of pattern completion (Rudy and O'Reilly 1999; Lee and Kesner 2002; Leutgeb et al. 2006; McHugh and Tonegawa 2009). In support of this idea, inactivation of the dorsal hippocampus with muscimol, a $\mathrm{GABA}_{\mathrm{A}}$ agonist, prior to the immediate-shock phase reduced freezing during the retention test (Matus-Amat et al. 2004). In contrast, infusion of AP-5 into dorsal hippocampus during the immediate-shock phase did not affect conditioning, as it did during the context preexposure phase (Matus-Amat et al. 2007). Together, the data argue that the rapid retrieval of a contextual representation is dependent on neural firing within the dorsal hippocampus, potentially through pattern completion, and that this activity is independent of NMDA receptor function. The lack of NDMA receptor dependence during context retrieval is consistent with SWRs, which underlie at least some forms of retrieval, mediated by AMPA receptor activation (Traub and Bibbig 2000; Maier et al. 2003, Colgin et al. 2004). This dissociation of signaling cascades during rapid retrieval suggests that the NDMA and AMPA receptors contribute to dis- tinct mnemonic processes which parse with IEG expression. Our findings illustrate that the neural activity associated with rapid retrieval is distinct from the neural activity required to activate IEG transcription during encoding. However, this assertion has to be further tested.

Given the convergence of data demonstrating that animals engage in rapid recall of a previously established contextual representation in CPFE, we conclude that IEG induction is not an accurate readout of rapid, pattern completion-based recall of a context memory. To the best of our knowledge, this is the first time that transcriptional activation of IEGs was examined specifically during a retrieval, and not an encoding, or "reencoding," event. This is significant as the cell activity findings imply that neural activity associated with rapid recall is different from that required for IEG induction, and by extension context encoding. The findings presented here add to a growing body of literature on the behavioral regulation of IEG induction, and the neural events associated with encoding and recall of context memory.

\section{Acknowledgments}

This work was supported by NSF Grant IOS 1026046 (J.F.G.). We thank Jessica Mendoza and Jennifer Czerniawski for helping with data acquisition/analysis and James McGaugh and Kazumasa Tanaka for critical comments on an earlier version of the manuscript.

\section{References}

Barot SK, Chung A, Kim JJ, Bernstein IL. 2009. Functional imaging of stimulus convergence in amygdalar neurons during Pavlovian fear conditioning. PLoS One 4: e6156.

Barrientos RM, O'Reilly RC, Rudy JW. 2002. Memory for context is impaired by injecting anisomycin into dorsal hippocampus following context exploration. Behav Brain Res 134: 299-306.

Blanchard RJ, Fukunaga KK, Blanchard DC. 1976. Environmental-control of defensive reactions to footshock. Bull Psychon Soc 8: 129-130.

Carr MF, Jadhav SP, Frank LM. 2011. Hippocampal replay in the awake state: a potential substrate for memory consolidation and retrieval. Nat Neurosci 14: $147-153$.

Carrive P. 2000. Conditioned fear to environmental context: cardiovascular and behavioral components in the rat. Brain Res 858: 440-445.

Colgin LL, Kubota D, Jia Y, Rex CS, Lynch G. 2004. Long-term potentiation is impaired in rat hippocampal slices that produce spontaneous sharp waves. J Physiol 558(Pt 3): 953-961.

Diba K, Buzsáki G. 2007. Forward and reverse hippocampal place-cell sequences during ripples. Nat Neurosci 10: 1241-1242. 
Fanselow MS. 1982. The postshock activity burst. Anim Learn Behav 10: $448-454$

Fanselow MS. 1986. Associative vs topographical accounts of the immediate shock-freezing deficit in rats: implications for the response selection rules governing species-specific defensive reactions. Learn Motiv 17: 16-39.

Fanselow MS. 1990. Factors governing one-trial contextual conditioning. Anim Learn Behav 18: 264-270.

Fanselow MS. 2000. Contextual fear, gestalt memories, and the hippocampus. Behav Brain Res 110: 73-81.

Foster DJ, Wilson MA. 2006. Reverse replay of behavioural sequences in hippocampal place cells during the awake state. Nature 440: $680-683$.

Good M, de Hoz L, Morris RGM. 1998. Contingent versus incidental context processing during conditioning: dissociation after excitotoxic hippocampal plus dentate gyrus lesions. Hippocampus 8: 147-159.

Girardeau G, Benchenane K, Wiener SI, Buzsáki G, Zugaro MB. 2009. Selective suppression of hippocampal ripples impairs spatial memory. Nat Neurosci 12: 1222-1223.

Guzowski JF. 2002. Insights into immediate-early gene function in hippocampal memory consolidation using antisense oligonucleotide and fluorescent imaging approaches. Hippocampus 12: 86-104.

Guzowski JF, McNaughton BL, Barnes CA, Worley PF. 1999. Environment-specific expression of the immediate-early gene Arc in hippocampal neuronal ensembles. Nat Neurosci 2: 1120-1124.

Guzowski JF, Lyford GL, Stevenson GD, Houston FP, McGaugh JL, Worley PF, Barnes CA. 2000. Inhibition of activity-dependent Arc protein expression in the rat hippocampus impairs the maintenance of long-term potentiation and the consolidation of long-term memory. $J$ Neurosci 11: 3993-4001.

Guzowski JF, Timlin JA, Roysam B, McNaughton BL, Worley PF, Barnes CA. 2005. Mapping behaviorally relevant neural circuits with immediate-early gene expression. Curr Opin Neurobiol 15: 599-606.

Guzowski JF, Miyashita T, Chawla MK, Sanderson J, Maes LI, Houston FP, Lipa P, McNaughton BL, Worley PF, Barnes CA. 2006. Recent behavioral history modifies coupling between cell activity and $\operatorname{Arc}$ gene transcription in hippocampal CA1 neurons. Proc Natl Acad Sci 103: $1077-1082$.

Hirsh R. 1974. The hippocampus and contextual retrieval of information from memory: a theory. Behav Biol 12: 421-444.

Jadhav SP, Kemere C, German PW, Frank LM. 2012. Awake hippocampal sharp-wave ripples support spatial memory. Science 336: 1454-1458.

Jones MW, Wilson MA. 2005. $\theta$ Rhythms coordinate hippocampalprefrontal interactions in a spatial memory task. PLoS Biol 3: e402.

Kiernan MJ, Westbrook RF. 1993. Effects of exposure to a to-be-shocked environment upon the rat's freezing response: evidence for facilitation, latent inhibition, and perceptual learning. Q J Exp Psychol B 46: 271-288.

Kubik S, Miyashita T, Guzowski JF. 2007. Using immediate-early genes to map hippocampal subregional functions. Learn Mem 14: 758-770.

Kudrimoti HS, Barnes CA, McNaughton BL. 1999. Reactivation of hippocampal cell assemblies: effects of behavioral state, experience, and EEG dynamics. J Neurosci 19: 4090-4101.

Lee I, Kesner RP. 2002. Differential contribution of NMDA receptors in hippocampal subregions to spatial working memory. Nat Neurosci 5: $162-168$

Leutgeb S, Leutgeb JK, Moser EI, Moser MB. 2006. Fast rate coding in hippocampal CA3 cell ensembles. Hippocampus 16: 765-774.

Lever C, Burton S, O'Keefe J. 2006. Rearing on hind legs, environmental novelty, and the hippocampal formation. Rev Neurosci 17: 111-133.

Liu X, Ramirez S, Pang PT, Puryear CB, Govindarajan A, Deisseroth K, Tonegawa S. 2012. Optogenetic stimulation of a hippocampal engram activates fear memory recall. Nature 484: 381-385.

Maier N, Nimmrich V, Draguhn A. 2003. Cellular and network mechanisms underlying spontaneous sharp wave-ripple complexes in mouse hippocampal slices. J Physiol 550(Pt 3): 873-887.

Maren S. 2001. Neurobiology of Pavlovian fear conditioning. Annu Rev Neurosci 24: 897-931.

Matus-Amat P, Higgins EA, Barrientos RM, Rudy JW. 2004. The role of the dorsal hippocampus in the acquisition and retrieval of context memory representations. J Neurosci 24: 2431-2439.

Matus-Amat P, Higgins EA, Sprunger D, Wright-Hardesty K, Rudy JW. 2007. The role of dorsal hippocampus and basolateral amygdala NMDA receptors in the acquisition and retrieval of context and contextual fear memories. Behav Neurosci 121: 721-731.
McHugh TJ, Tonegawa S. 2009. CA3 NMDA receptors are required for the rapid formation of a salient contextual representation. Hippocampus 19: $1153-1158$.

Messaoudi E, Kanhema T, Soulé J, Tiron AJ, Dagyte G, da Silva B, Bramham CR. 2007. Sustained Arc/Arg3.1 synthesis controls long-term potentiation consolidation through regulation of local actin polymerization in the dentate gyrus in vivo. J Neurosci 27: $10445-10455$

Miyashita T, Kubik S, Haghighi N, Steward O, Guzowski JF. 2009. Rapid activation of plasticity-associated gene transcription in hippocampal neurons provides a mechanism for encoding of one-trial experience. $J$ Neurosci 29: 898-906.

Moriss DC, Bransford JD, Franks JJ. 1977. Levels of processing versus transfer appropriate processing. J Verb Learn Verb Behav 16: 519-533.

Nalloor R, Bunting KM, Vazdarjanova A. 2012. Encoding of emotionpaired spatial stimuli in the rodent hippocampus. Front Behav Neurosci 6: 27.

Nomura H, Nonaka A, Matsuki N. 2012. Population activity in the dorsal hippocampal CA1 encoding the surrounding environment is absent during contextual fear memory expression. Neuroscience 220: $19-25$.

Norman KA, O'Reilly R. 2003. Modeling hippocampal and neocortical contributions to recognition memory: a complementary-learningsystems approach. Psychol Rev 110: 611-646.

Pevzner A, Miyashita T, Schiffman AJ, Guzowski JF. 2012. Temporal dynamics of Arc gene induction in hippocampus: relationship to context memory formation. Neurobiol Learn Mem 97: 313-320.

Pfieffer BE, Foster DJ. 2013. Hippocampal place-cell sequences depict future paths to remembered goals. Nature 497: 74-79.

Ramirez S, Liu X, Lin PA, Suh J, Pignatelli M, Redondo RL, Ryan TJ, Tonegawa S. 2013. Creating a false memory in the hippocampus. Science 341: $387-391$.

Rolls ET, Kesner RP. 2006. A computational theory of hippocampal function, and empirical tests of the theory. Prog Neurobiol 79: 1-48.

Rudy JW, O'Reilly RC. 1999. Contextual fear conditioning, conjunctive representations, pattern completion, and the hippocampus. Behav Neurosci 113: 867-880.

Rudy JW, O'Reilly RC. 2001. Conjunctive representations, the hippocampus, and contextual fear conditioning. Cogn Affect Behav Neurosci 1: 66-82.

Rudy JW, Barrientos RM, O'Reilly RC. 2002. Hippocampal formation supports conditioning to memory of a context. Behav Neurosci 116: 530-538.

Rudy JW, Huff NC, Matus-Amat P. 2004. Understanding contextual fear conditioning: insights from a two-process model. Neurosci Biobehav Rev 28: $675-685$.

Tanaka KZ, Wiltgen BJ. 2013. Selective silencing of previously activated hippocampal neurons. In 2013 Neuroscience meeting planner, program no. 94.07/III7. Society for Neuroscience, San Diego, CA.

Teyler TJ, DiScenna P. 1986. The hippocampal memory index theory. Behav Neurosci 100: 147-154.

Teyler TJ, Rudy JW. 2007. The hippocampal indexing theory and episodic memory: updating the index. Hippocampus 17: 1158-1169.

Tolman EC. 1925. Purpose and cognition: the determiners of animal learning. Psychol Rev 32: 285-297.

Tolman EC, Ritchie BF, Kalish D. 1946. Studies in spatial learning. 1. Orientation and the short-cut. J Exp Psychol 36: 13-24.

Traub RD, Bibbig A. 2000. A model of high-frequency ripples in the hippocampus based on synaptic coupling plus axon-axon gap junctions between pyramidal neurons. J Neurosci 20: 2086-2093.

Vazdarjanova A, Guzowski JF. 2004. Differences in hippocampal neuronal population responses to modifications of an environmental context: evidence for distinct, yet complementary, functions of CA3 and CA1 ensembles. J Neurosci 24: 6489-6496.

Westbrook RF, Good AJ, Kiernan MJ. 1994. Effects of the interval between exposure to a novel environment and the occurrence of shock on the freezing responses of rats. Q J Exp Psychol B 47: 427-446.

Wiltgen BJ, Zhou M, Cai Y, Balaji J, Karlsson MG, Parivash SN, Li W, Silva AJ. 2010. The hippocampus plays a selective role in the retrieval of detailed contextual memories. Curr Biol 20: 1336-1344.

Wiltgen BJ, Wood AN, Levy B. 2011. The cellular mechanisms of memory are modified by experience. Learn Mem 18: 747-750.

Received April 15, 2014; accepted in revised form October 6, 2014. 


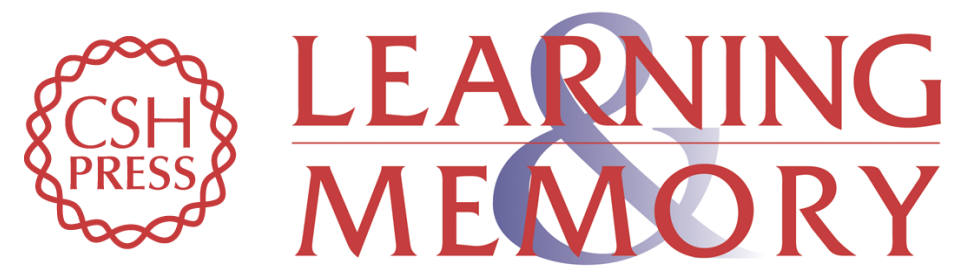

\section{Immediate-early gene transcriptional activation in hippocampus CA1 and CA3 does not accurately reflect rapid, pattern completion-based retrieval of context memory}

Aleksandr Pevzner and John F. Guzowski

Learn. Mem. 2015, 22:

Access the most recent version at doi:10.1101/lm.035469.114

References This article cites 57 articles, 11 of which can be accessed free at: http://learnmem.cshlp.org/content/22/1/1.full.html\#ref-list-1

Creative This article is distributed exclusively by Cold Spring Harbor Laboratory Press for the Commons first 12 months after the full-issue publication date (see

License http://learnmem.cshlp.org/site/misc/terms.xhtml). After 12 months, it is available under a Creative Commons License (Attribution-NonCommercial 4.0 International), as described at http://creativecommons.org/licenses/by-nc/4.0/.

Email Alerting Receive free email alerts when new articles cite this article - sign up in the box at the Service top right corner of the article or click here. 